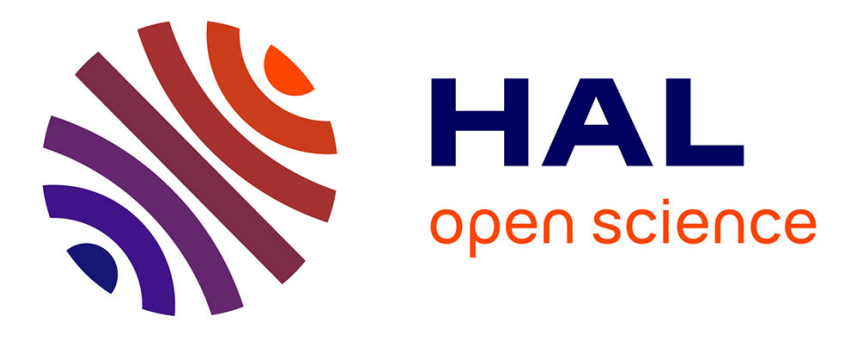

\title{
Multi-lag Phase Diagram Analysis for Transient Signal Characterization
}

\author{
Cindy Bernard, Angela Digulescu, Alexandre Girard, Cornel Ioana
}

\section{To cite this version:}

Cindy Bernard, Angela Digulescu, Alexandre Girard, Cornel Ioana. Multi-lag Phase Diagram Analysis for Transient Signal Characterization. Charles L. Webber, Jr., Cornel Ioana, Norbert Marwan. Recurrence Plots and Their Quantifications: Expanding Horizons, 180, Springer, pp.39-63, 2016, Springer Proceedings in Physics, 978-3-319-29921-1. 10.1007/978-3-319-29922-8_3 . hal-01448224

\section{HAL Id: hal-01448224 \\ https://hal.science/hal-01448224}

Submitted on 27 Jan 2017

HAL is a multi-disciplinary open access archive for the deposit and dissemination of scientific research documents, whether they are published or not. The documents may come from teaching and research institutions in France or abroad, or from public or private research centers.
L'archive ouverte pluridisciplinaire $\mathbf{H A L}$, est destinée au dépôt et à la diffusion de documents scientifiques de niveau recherche, publiés ou non, émanant des établissements d'enseignement et de recherche français ou étrangers, des laboratoires publics ou privés. 


\title{
Multi-lag phase diagram analysis for transient signal characterization
}

Cindy Bernard, Angela Digulescu, Alexandre Girard and Cornel Ioana

\begin{abstract}
Phase diagram analysis is a potential technique that can offer interesting information regarding the signal's shapes and eventually transient signal characterization. Indeed by choosing wisely the lag in phase diagram representations, it is possible to highlight mathematical properties such as time-shift and time-scale operators, as well as amplitude modifications. Therefore, this paper develops the concept of multi-lag phase diagram analysis (MLPDA), as well as different methods aimed to extract parsimonious parameters from signal's phase diagrams calculated for different values of lags. By combining all of them, we are then able to explore new ways of transient signal characterization.
\end{abstract}

\section{Introduction}

In this chapter, we only consider transient signals that are characterized by sudden amplitude changes. They usually traduce mechanical, electrical, or electromagnetic phenomena that are very important to monitor. Such signal can be given by:

$$
s(t)=A(t), t \in\left[t_{0}, t_{0}+D\right]
$$

Cindy Bernard

Grenoble-INP, Gipsa-lab, Grenoble, France, e-mail: cindy.bernard@ gipsa-lab.fr

Angela Digulescu

Name, Address of Institute e-mail: name@email.address

Alexandre Girard

EDF R\&D, Chatou, France e-mail: Alexandre.girard@edf.fr

Cornel Ioana

Grenoble-INP, Gipsa-lab, Grenoble, France, e-mail: cornel.ioana@gipsa-lab.fr 
where the duration $D$ is assumed much shorter than the signal's observation time, and $t_{0}$ the starting time of the signal. The amplitude function $A(t)$ of the signal is assumed to have high order derivatives.

We particularly focus on the case of transients observed at a given distance from the source and that consists of not only the transient at the origin (as it is generated by the phenomenon) but also of the propagation and the receiver processing effects. Thus, the deformations introduced by the propagation are of great interest to characterize the environment.

The methods proposed in our work will contribute to offer a potential interesting solution, attempting to provide a general analyis framework of such signals. To do so, we propose to investigate the signals similarities and dissimilarities through recurrence plot (RP) which has been introduced by Eckmann et al. [1] in 1987 in order to visualize recurrences of higher-dimensional phase space trajectories in nonlinear data time series. By recurrence, we refer to the return of a state of a system to a previously visited point. Recurrence Plot Analysis (RPA) has then been derived from RP and is based on three major steps: time-delay embedding (1980-1981) [2, 3], recurrence plots (1987) and recurrence quantification analysis (RQA)(1992-2002) $[4,5]$. More details about the history of RPA can be found in [6] and an international website gathers all the advances and communications of the community [7].

Therefore, the main idea of this chapter is to investigate the different signals's properties in their phase diagrams in order to explore the similarities and dissimilarities between them. To do so, Section 2 first provides different mathematical properties such as time-scale transformations and amplitude modifications between transients that can be highlighted by the lag diversity in phase diagrams. Then Section 3 proposes five new descriptors for transient characterization that enables to extract the previously cited transformations. An application example is then presented in Section 4. Finally, Section 5 provides some conclusions and perspectives of work.

\section{Mathematical properties of lag diversity in phase diagram}

In order to illustrate the concept of multi-lag PDA, a transient is modeled as a modulated cosine $s(n)$ defined for $n \in\{1, \ldots, N\}$ such as:

$$
s(n)=\left\{\begin{array}{c}
\cos \left(2 \pi f_{0} n\right) w(n) \text { if } n \in\left[n_{0}, n_{0}+\Delta\right] \\
0 \text { otherwise }
\end{array}\right.
$$

with $w(n)$ a modulating window (such as a Hanning window), $f_{0}$ the central frequency of the modulation (we consider $f_{0}=3$ in our examples), $n_{0}$ the beginning of the transient and $\Delta$ the transient duration.

Phase space diagram analysis of signal $s(n)$, using the embedding dimension $m$ and lag $\tau$, corresponds to a representation $T$ that is defined as follows: 


$$
\begin{aligned}
T: \mathbb{R}^{N} \times \mathbb{N} \times \mathbb{N} & \longrightarrow \mathscr{M}_{N-(m-1) \tau, m}(\mathbb{R}) \\
(s, \tau, m) & \longmapsto T_{m, \tau}(s)
\end{aligned}
$$

where:

$$
T_{m, \tau}(s)=\left(\begin{array}{cccc}
s(1) & \ldots & s(1+(j-1) \tau) & \ldots s(1+(m-1) \tau) \\
s(2) & \ldots & s(2+(j-1) \tau) & \ldots s(2+(m-1) \tau) \\
\vdots & & & \\
s(i) & \ldots & s(i+(j-1) \tau) & \ldots s(i+(m-1) \tau) \\
\vdots & & &
\end{array}\right)
$$

which can be summarized by:

$$
T_{m, \tau}(s)=\left\{t_{i, j}\right\}_{i=\{1,2 \ldots, N-(m-1) \tau\}, j=\{1,2, \ldots, m\}}
$$

with:

$$
t_{i, j}=s(i+(j-1) \tau)
$$

Each row of the $T$ matrix corresponds to a phase space vector. Each column corresponds to the coordinates of one axis of the phase space.

Let us now investigate the properties of this representation to the time-shift operator, the scale operator and amplitudes changes. We define three signals $s_{1}(n), s_{2}(n)$ and $s_{3}(n)$ such as:

$$
\begin{aligned}
& s_{1}(n)=s(n+\delta) \\
& s_{2}(n)=s(\alpha n) \\
& s_{3}(n)=\beta s(n)
\end{aligned}
$$

with $\delta$ the time-shift delay, $\alpha \in \mathbb{R}^{+}$the dilation coefficient and $\beta$ an amplitude modification coefficient. We propose to illustrate the different properties with equations and numerical examples that are shown in Figure 1. The numerical examples are computed with the following parameters: $\delta=55, \alpha=2$ and $\beta=1.5$.

\section{Investigation of the time-shift invariance property}

We can start by considering the phase space vector of $s_{1}(n)$ at instant $n$ and the relation given by Eq. 7 . We have:

$$
\begin{aligned}
{\left[s_{1}(n), s_{1}(n+\tau), \ldots, s_{1}(n+(m-1) \tau)\right] } & =[s(n+\delta), s(n+\delta+\tau), \ldots, s(n+\delta+(m-1) \tau)] \\
& =\left[s\left(n_{0}\right) s\left(n_{0}+\tau\right), \ldots, s\left(n_{0}+(m-1) \tau\right)\right]
\end{aligned}
$$


(a)

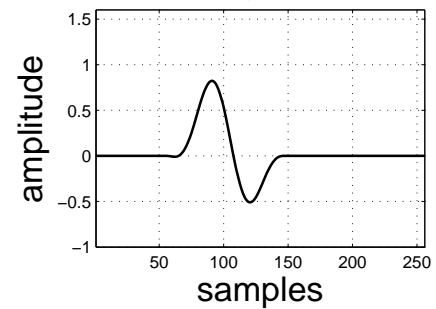

(c)

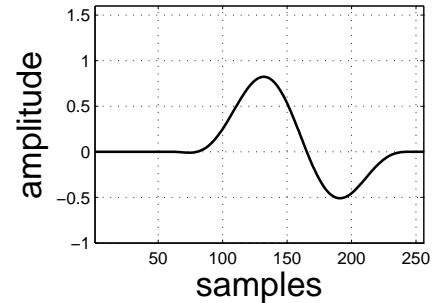

(b)

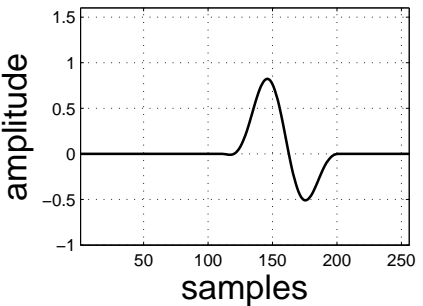

(d)

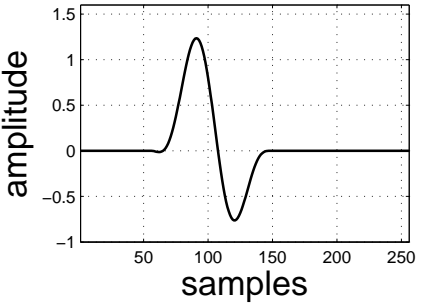

Fig. 1 Temporal data of: (a) $s(n)$, (b) $s_{1}(n)$, (c) $s_{2}(n)$ and (d) $s_{3}(n)$

This result states that even if two identical signals are time-shifted, their phase space diagrams are invariant. Mathematically speaking, it means that the rows of $T_{m, \tau}\left(s_{1}\right)$ are the same as $T_{m, \tau}(s)$ 's but they suffered the circular permutation and are shifted compared to $T_{m, \tau}(s)$. This property is illustrated by Figure 2 where we can see that for $m=2$ and $\tau=8$ the phase space diagrams of the two signals are exactly the same despite the time-shift between the signals.

(a)

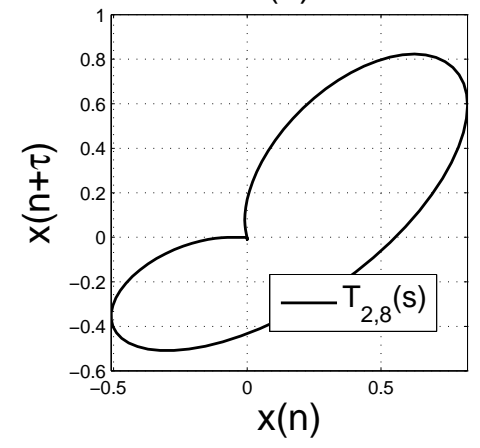

(b)

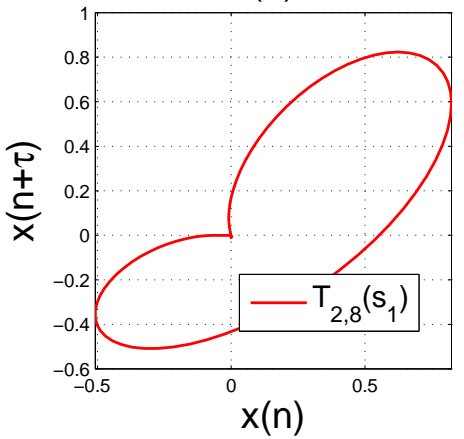

Fig. 2 (a) $T_{2,4}(s)$ and (b) $T_{2,4}\left(s_{1}\right)$ phase space diagrams. The trajectories are the same despite the time-shift. 


\section{Investigation of the time-scaling property}

We now consider $s_{1}(n)$ 's phase space vector at instant $n$ and the relation given by Eq. 8. We have:

$$
\begin{aligned}
{\left[s_{2}(n), s_{2}(n+\tau), \ldots, s_{2}(n+(m-1) \tau)\right] } & =\left[s\left(\alpha n+n_{0}\right), \ldots, s\left(\alpha(n+(m-1) \tau)+n_{0}\right)\right] \\
& =\left[s\left(n_{1}\right), s\left(n_{1}+\alpha \tau\right), \ldots, s\left(n_{1}+(m-1) \alpha \tau\right)\right] \\
& =\left[s\left(n_{1}\right), s\left(n_{1}+\tau_{0}\right), \ldots, s\left(n_{1}+(m-1) \tau_{0}\right)\right]
\end{aligned}
$$

This result states that for a given $m$ there exists many sets of lags $[\tau, \alpha \tau]$ that enable an invariance of phase space diagrams. It means that it is possible to identify two signals that are related by a dilation. Figure 3 presents the phase space diagrams of $s(n)$ and $s_{1}(n)$ for two differents sets of lags: [4,4] and [4,8]. For the first set, the two diagrams do not overlap, whereas the second set enables a perfect superposition of the two diagrams.

(a)

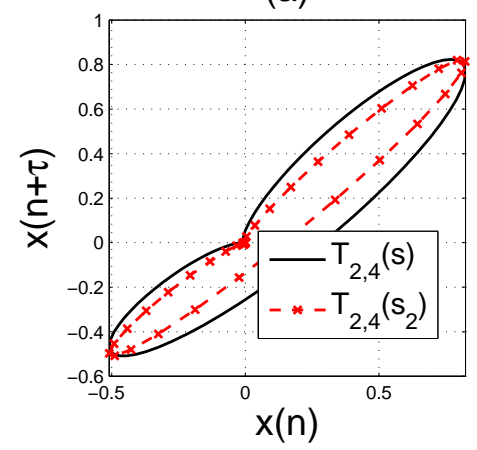

(b)

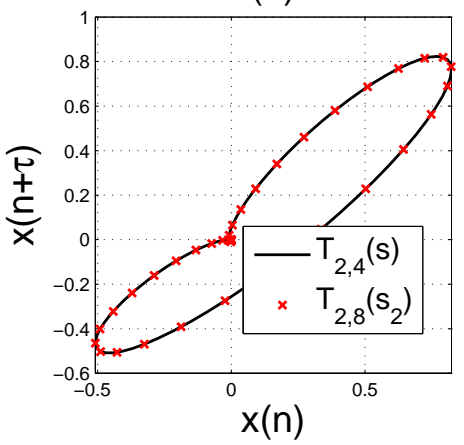

Fig. 3 Phase space diagrams of $s(n)$ and $s_{2}(n)$ for $m=2$ and different sets of lags: (a) $[4,4]$ and (b) $[4,8]$. The dilation connection between the two signals can be enlightened by using the appropriate set of lags.

\section{Investigation of the amplitude coefficient modification}

Finally, we consider $s_{3}(n)$ 's phase space vector at instant $n$ and the relation given by Eq. 9 . We have:

$$
\begin{aligned}
{\left[s_{3}(n), s_{3}(n+\tau), \ldots, s_{3}(n+(m-1) \tau)\right] } & =[\beta s(n), \beta s(n+\tau), \ldots, \beta s(n+(m-1) \tau)] \\
& =\beta[s(n), s(n+\tau), \ldots, s(n+(m-1) \tau)]
\end{aligned}
$$

which can be summarized by: 


$$
T_{m, \tau}\left(s_{3}\right)=\beta T_{m, \tau}(s)
$$

This result shows that the amplitude change is equivalent, in the phase space diagram representation, with a shape-invariant scale transformation. This is illustrated by Figure 4.

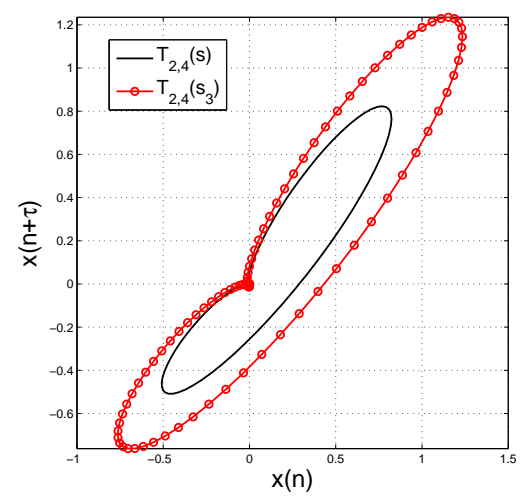

Fig. $4 T_{2,4}\left(s_{3}\right)$ and $T_{2,4}(s)$ phase space diagrams. They are related by a shape-invariant scale transformation.

\section{Multi-lag phase diagram analysis}

The previous section has shown that phase diagram representations can potentially be invariant to the main transforms of signals such as: time-shift, time-scale changes, amplitude modification, etc... The key point of the multi-lag based representation is that the invariance can be controlled by the lag choice, which makes possible a better exploration of the anayzed signals.

In this section, we propose different descriptors to extract parsimonious parameters from each diagram acquired for a given lag. The evolution of these descriptors with respect to the lags is then explored conducting to new representation tools for transients.

In this Chapter, we restrained ourselves to $m=2$ in order to visualize the results but the work can be extended to higher embedding dimension. We also note $y(n)$ for $x(n+\tau)$ in order to simplify the notations. 


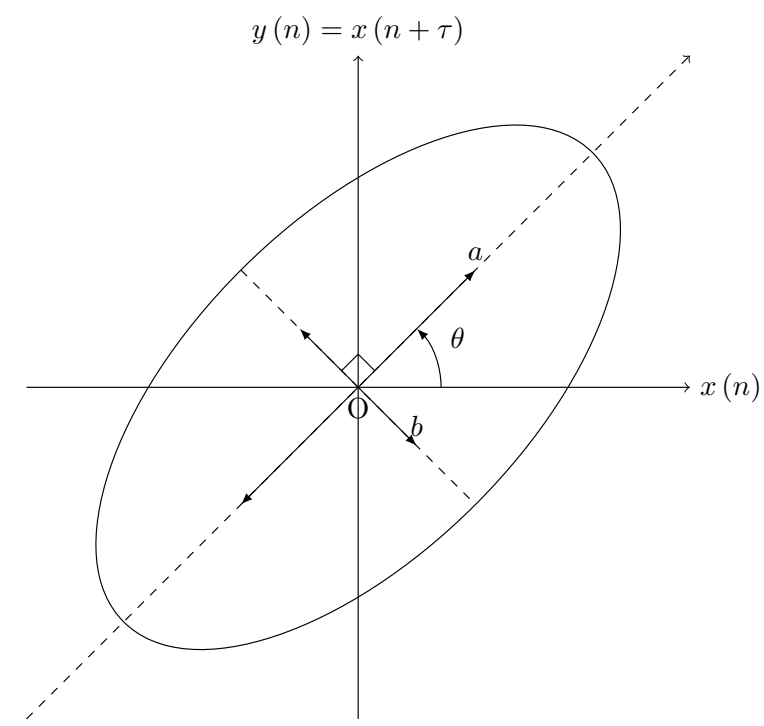

Fig. 5 Ellipse modeling: major and minor axis are determined by an SVD on phase space diagram, and a least square fitting method is used to calculate $a$ and $b$.

\subsection{Ellipse modeling}

Generally, signals can have various trajectories with different shapes, but in this subsection, we propose a general model for approximation of trajectories based on ellipse shape (Figure 5). This choice is quite natural since the ellipse model is specific to harmonic signals. Therefore, the model is simple and enables to extract three parameters per phase space diagrams:

- the polar angle $\theta$ of the ellipse assuming that $\theta$ is the angle between the first axis and the major semi-axis

- the major semi-axis $a$

- the minor semi-axis $b$

We assume the center of the ellipse being the center of phase space diagrams as transients can be considered as zero-mean signals.

The first step consists in performing a singular value decomposition (SVD) of the phase space diagram to calculate its eigenvectors. Those are of great interest as they define a new basis that reflects the distribution of the data and also correspond to the major and minor axis of the ellipse model. Then, a least squares fitting method is performed to determine the ellipse that satisfy the following equation in the new basis defined by the eigenvectors:

$$
A x^{2}+B y^{2}=1
$$

where the semi-major and semi-minor axis are given by: 


$$
\left\{\begin{array}{l}
a=1 / \sqrt{A} \\
b=1 / \sqrt{B}
\end{array}\right.
$$

At this point of the study, each diagram is modeled by an ellipse with these three parameters $[a, b, \theta]$. The evolution of $\theta$ enables to estimate an apparent periodicity of the transient, while the evolution of $a$ and $b$ enables to know how the data is distributed in the phase space. If they are scattered over a large area, it means that the lag used to construct the representation is not representative of the transient contruction. On the other hand, if they are rather confined into a smaller area, it means that the lag is representative and is well adapted to the study of this transient.
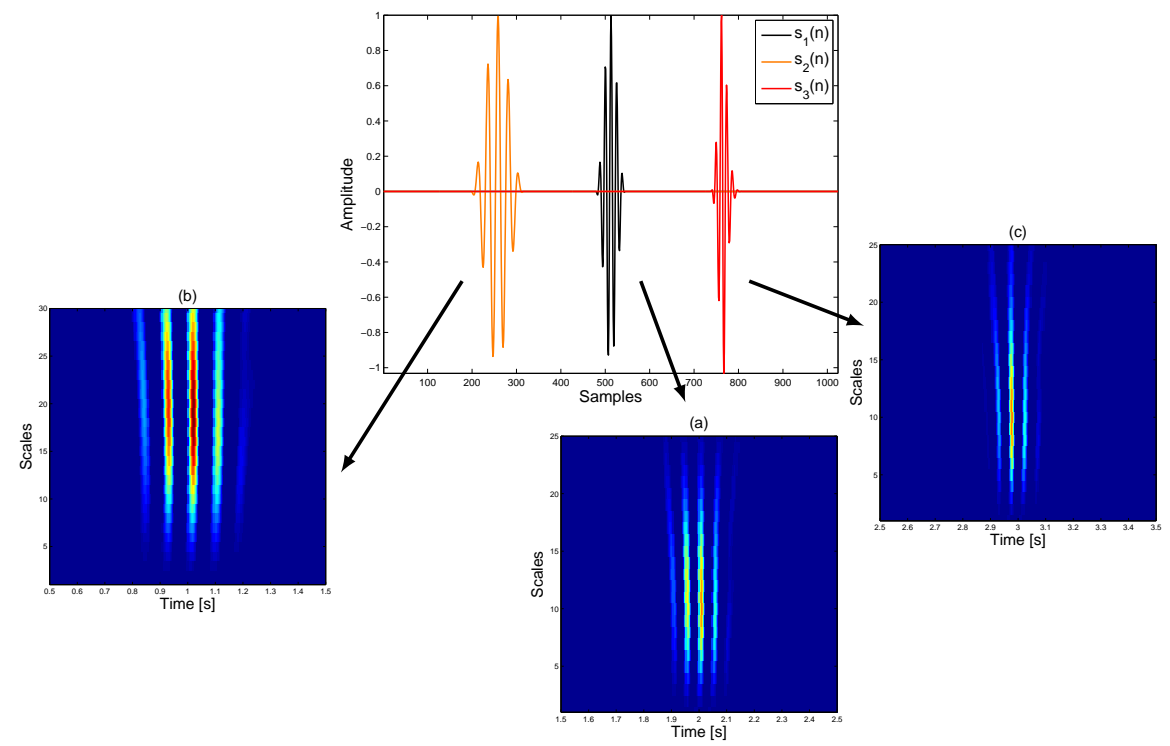

Fig. 6 Temporal signals studied in this section and its wavelet transforms using the Daubechies mother wavelet.

In order to illustrate the method, let consider three signals $s_{1}(n), s_{2}(n)$ and $s_{3}(n)$ that are modulated cosines. $s_{1}(n)$ and $s_{2}(n)$ are related by a time-scale transformation while $s_{3}(n)$ is the result of a 10-th low-pass FIR digital filtering applied on $s_{1}(n)$ with the normalized cutoff frequency of 0.1 . Temporal signals are shown in Figure 6. At first look, it is quite difficult to tell appart $s_{1}(n)$ from $s_{3}(n)$ and a time-scale analysis would not do better as their frequency contents are really close (Figure 6).

For the three signals and $\tau \in\{1, \ldots, 30\}$, we model each phase diagram by an ellipse and record the variation of $a, b$ and $\theta$ with respect to the lags. As we can see in Figures 7 and 8, the 3 parameters present an apparent periodicity that are related to the apparent periodicities of the signals. We call apparent periodicity the number of samples between two successive zero-crossing. As an example, the number of 


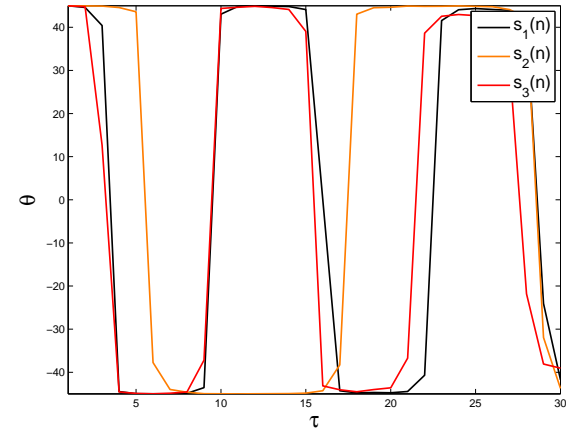

Fig. 7 Evolution of $\theta$ with respect of $\tau$ for the three studied signals.

(a)

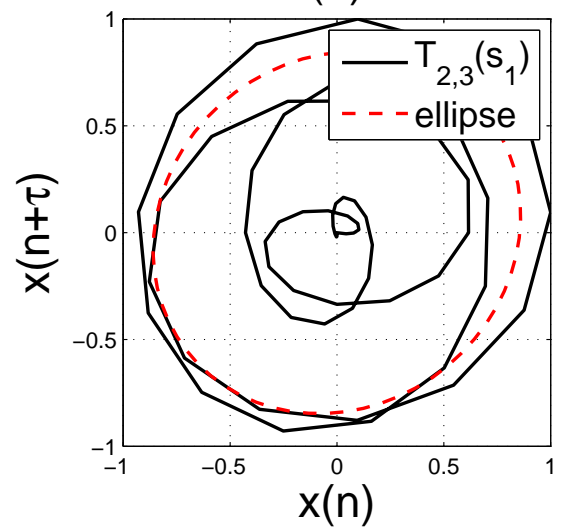

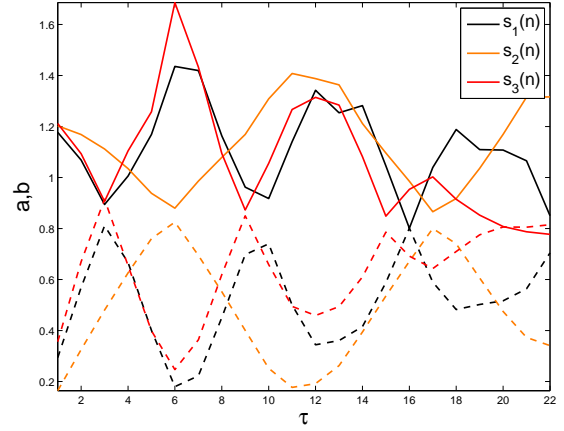

Fig. 8 Evolution of $a$ (continuous line) and $b$ (dashed line) with respect of $\tau$ for the 3 studied signals.

(b)

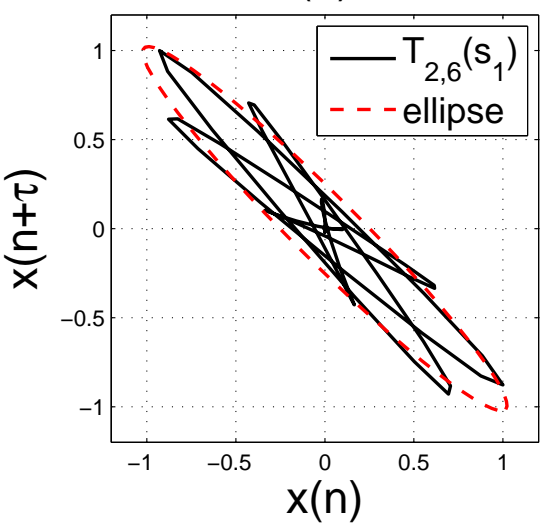

Fig. 9 (a) $T_{2,3}\left(s_{1}\right)$ phase space diagram and its associated ellipse model. (b) $T_{2,6}\left(s_{1}\right)$ phase space diagram and its associated ellipse model.

lags between two successive maxima of $\theta$ is equal to 13 for $s_{1}(n), 23$ for $s_{2}(n)$ and 12 for $s_{3}(n)$, while the apparent periodicity is of 12.5 samples for $s_{1}(n), 22.25$ for $s_{2}(n)$ and 11.75 for $s_{3}(n)$ which is coherent with the previous values.

The periodicity of $a$ and $b$ corresponds to the half period of the signals (Figure 8). Their maximal values correspond to phase diagrams that can be modeled by circles (same value for both parameters), meaning that the phase diagrams are more scattered in the phase space. This can be seen in Figure 9 where we plotted $s_{1}(n)$ 's phase diagrams for $m=2$ and $\tau=\{3,6\}$. For $\tau=3$ that enables to obtain a maximal value for $b$, the phase diagram can be modeled by a circle and is well distributed in the space, while for $\tau=6$, the phase diagram is concentrated into a smaller area.

We have seen that with the ellipse modeling it was posssible to summarize each representation by only three parameters and estimate the apparent frequency of a 
transient. We can also explore the distribution of the diagram in the phase space which is of great interest as it enables to highlight the lags that provide a great concentration of the data or on the contrary a dispersal.

\subsection{Trend modeling}

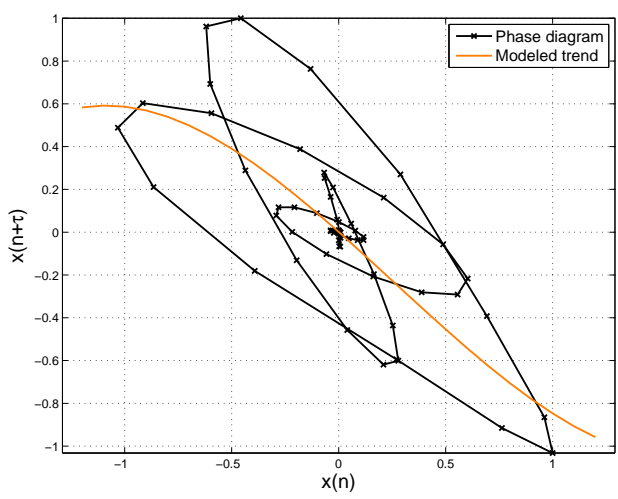

Fig. 10 Phase diagram's trend is modeled by a third degree polynomial.

In the previous subsection, we have seen that phase diagrams were more or less concentrated around a line that tends to rotate around the origin of the phase space (due to the zero-mean of the modeled signals). This is why, we want to model this line as a third degree polynomial (Figure 10) in order to quantify the rotation and the natural trend of the diagram [8]. The model is defined as follows:

$$
y=\widehat{a} x^{3}+\widehat{b} x^{2}+\widehat{c} x+\widehat{d}
$$

To do so, we consider the diagram as a scatterplot and perform a least square fitting estimation by minimizing the following sum:

$$
\underset{\widehat{a}, \widehat{b}, \widehat{c}, \widehat{d}}{\operatorname{Argmin}} \sum_{i=1}^{N}\left(s(i+\tau)-\left(\widehat{a} s^{3}(i)+\widehat{b} s^{2}(i)+\widehat{c s}(i)+\widehat{d}\right)\right)
$$

where $s(i)$ corresponds to the analyzed signal for $i \in\{1, \ldots, N\}$; $\mathrm{N}$ being the number of samples.

Therefore, for each representation, phase diagrams are summarized by four parameters $\left\{\widehat{a}_{\tau}, \widehat{b}_{\tau}, \widehat{c}_{\tau}, \widehat{d}_{\tau}\right\}$ that vary with respect to $\tau$. As studied signals can always be considered as zeros mean, we can remove from consideration $\widehat{d}$ : this parameter will always be equal to zero. Thus, three parameters remain: $\widehat{a}, \widehat{b}$ and $\widehat{c}$. They enable 
to discriminate transients by looking at their evolutions with respect to $\tau$ and they also permit to highlight similitudes by looking at one parameter with respect to another. This last representation allows to get rid of the evolution of $\tau$ and investigate if whether or not the diagrams have similar trends for different lags: this can reflects a time-scaling operation.
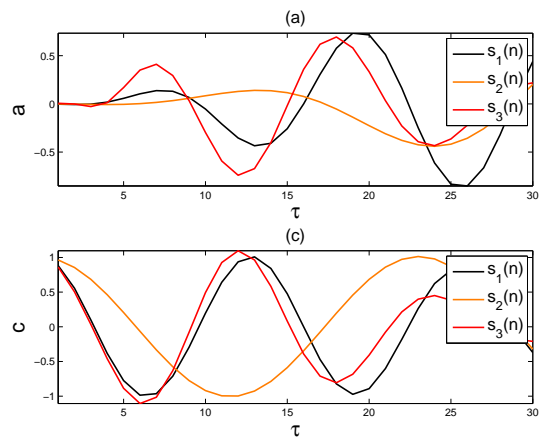
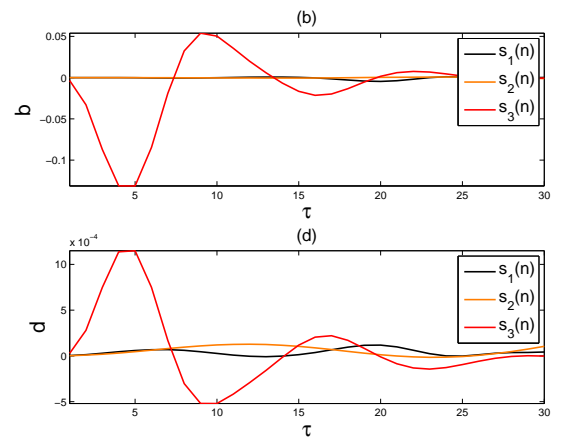

Fig. 11 Evolution of (a) $\widehat{a}$,(b) $\widehat{b}$, (c) $\widehat{c}$ and (d) $\widehat{d}$ with respect of $\tau$.

To illustrate the concept of trend modeling, we consider the three signals that were introduced in the previous subsection. For all of them and $\tau=\{1, \ldots, 30\}$, we model each phase diagram's trend by a third degree polynomial and record the variations of the four parameters with respect to the lag. As we can see in Figure 11, it is quite easy to discriminate between $s_{1}(n)$ and $s_{3}(n)$ by looking at the evolution of the parameters of interest. The apparent periodicity of the parameters corresponds to the apparent periodicity of the signals. As an example, the number of lags between two successive maxima of $\widehat{c}$ is equal to 12 for $s_{1}(n), 22$ for $s_{2}(n)$ and 11 for $s_{3}(n)$, while the apparent periodicity is of 12.5 samples for $s_{1}(n), 22.25$ for $s_{2}(n)$ and 11.75 for $s_{3}(n)$ which is coherent with the theoretical values. We can also notice that the evolution of $\widehat{d}$ 's is very small (below 0.0001), as well as for the evolution of parameter $\widehat{b}$. This is why we remove this last parameter from consideration as well.

Figure 12 presents the evolution of $\widehat{c}$ with respect to the evolution of $\widehat{a}$. This representation is interesting as we get rid of the evolution of $\tau$. It enables to highlight signals that would have the same phase diagram's trends for different values of lags. This is the case for $s_{1}(n)$ and $s_{2}(n)$ where the evolution of $[\widehat{a}, \widehat{c}]$ for both signals overlap in the representation. This is coherent as the two signals are related by a time-scale operator. As $s_{1}(n)$ and $s_{3}(n)$ are related by a low-pass filtering, we can see that even if their representations are similar, they do not overlap. This shows the complexity introduced by the filtering.

The modeling of phase diagrams's trend by a third degree polynomial is of great interest as it enables to detect if transients are related by a time-scale transformation. However, at this point of the study, there is no method that tells us what couples of lags we should use to highlight this transformation. This is why we moved torward a matching phase diagram technique that is presented in the next subsection. 
(a)

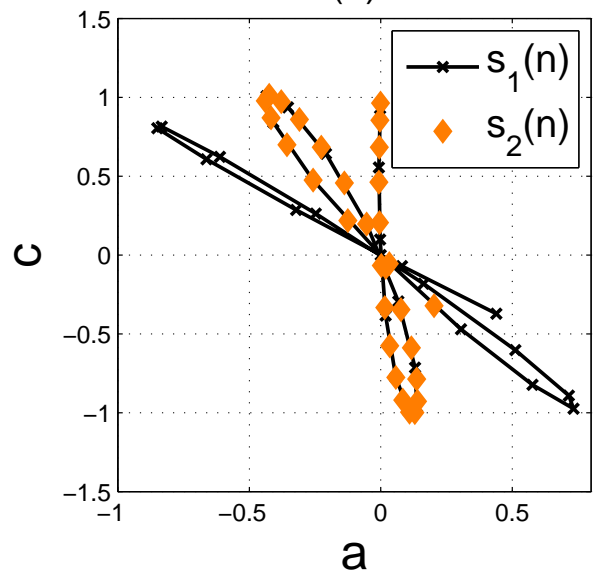

(b)

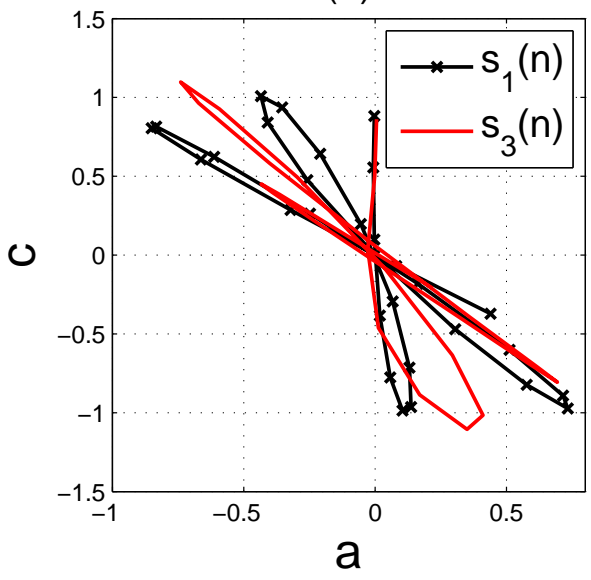

Fig. 12 Evolution of $\widehat{c}$ with respect to $\widehat{a}$ for the 3 studied signals.

\subsection{Extremum points / bounding box}
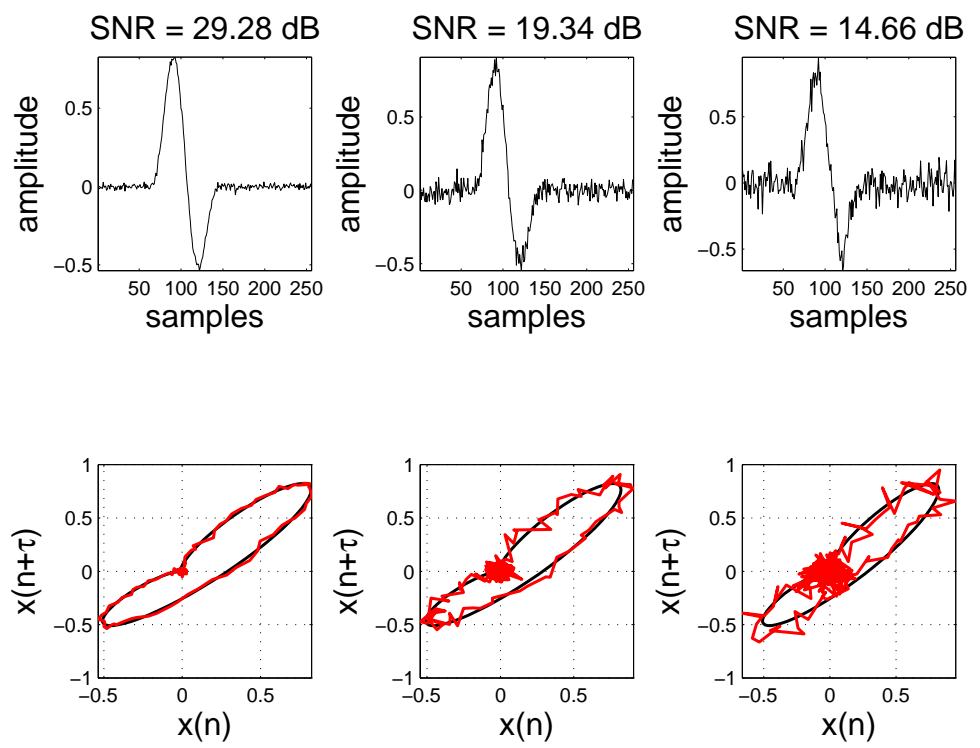

Fig. 13 Noisy transients and their respective phase diagrams for different level of noise. 
Generally, when we talk about SNR for transient analysis, we only consider signal and noise over the duration of the transient. Thus, the SNR is defined as follows:

$$
\mathbf{S N R}_{d B}=10 \log _{10} \frac{\sum_{n} s(n)}{\sum_{n} b(n)}
$$

where $s(n)$ corresponds to the noise-free transient and $b(n)$ to the noise, defined for $n \in \Delta$ with $\Delta$ being the time interval where is defined the transient of interest.

When it comes to noise in phase diagram, we can see in Figure 13 that the external contour remains more or less the same depending on the level of noise considered. This is the reason why we focus on the bounding box of the trajectory that is delimited by the maximal and minimal values of the studied signal.

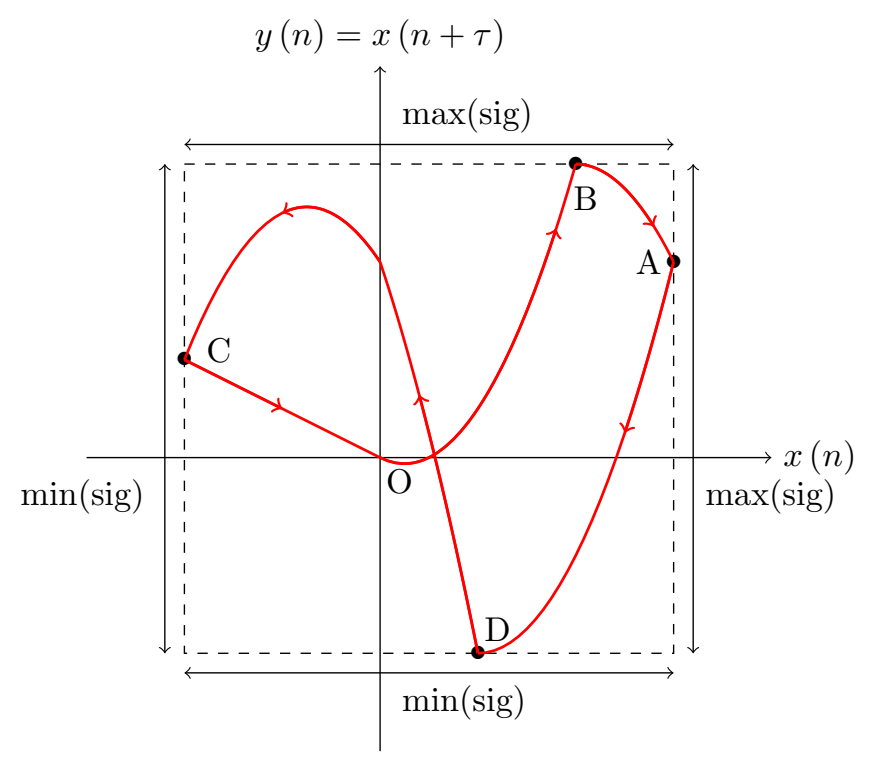

Fig. 14 Bounding box that confines transient trajectories are delimited by the maximal and minimal values of transients.

We thus define 4 remarkables coordinates defined as follows and illustrated in Figure 14:

$$
\begin{aligned}
& A:\left\{\begin{array}{l}
\forall n, \quad x\left(n_{1}\right)=\max _{n}(x(n)) \\
y\left(n_{1}\right)
\end{array}\right. \\
& B:\left\{\begin{array}{l}
x\left(n_{2}\right) \\
\forall n, \quad y\left(n_{2}\right)=\max _{n}(y(n))
\end{array}\right. \\
& C:\left\{\begin{array}{l}
\forall n, \quad x\left(n_{3}\right)=\min _{n}(x(n)) \\
y\left(n_{3}\right)
\end{array}\right.
\end{aligned}
$$




$$
D:\left\{\begin{array}{l}
x\left(n_{4}\right) \\
\forall n, \quad y\left(n_{4}\right)=\min _{n}(y(n))
\end{array}\right.
$$

In order to compare two transient signals analysis, we consider the signals' phase space diagrams for different values of lag $\left[\tau_{1, i}, \tau_{2, i}\right]_{i \in\left[1, \ldots, \tau_{\max }\right]}$ and we look for their extremum points $\left[A_{k, i}, B_{k, i}, C_{k, i}, D_{k, i}\right]_{k=1,2}$. Then, we compute 4 matrices $H_{A}, H_{B}$, $H_{C}$ and $H_{D}$ defined as follows:

$$
\begin{aligned}
H_{X} & =\left\{h_{X, i, j}\right\}_{i, j \in\left[1, \ldots, \tau_{\max }\right]} \\
& =\left\{\mathscr{D}\left(X_{1, i}, X_{2, j}\right)\right\}
\end{aligned}
$$

where $\mathscr{D}$ describes a given metric and $X$ the extremum point taken into consideration.

Each matrix provides a "map" of the distances between the extremum points of each phase space diagrams calculated for different values of lags. Therefore, it enables to discover which couples of lags $\left[\tau_{1}, \tau_{2}\right]$ need to be used to provide a match between two extremum points.

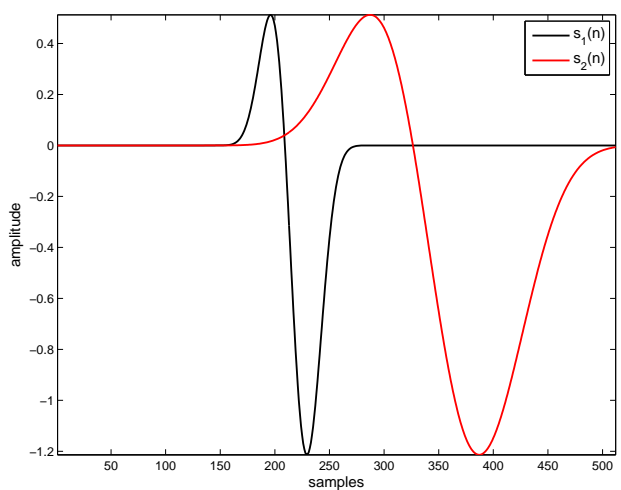

Fig. 15 Temporal data of the studied signals

In order to illustrate this concept, let consider two transients related by a timescale transformation with the dilation coefficient $\alpha=3$. Temporal data presented in Figure 15 shows that $s_{2}(n)$ (in red) is only dilated compared to $s_{1}(n)$ (in black) and does not suffer amplitude changes. In this part, the purpose is to highlight the time-scale relation between these signals.

We first calculate the 4 matrices described previously using the Euclidean norm $\mathscr{D}_{2}$ defined as follows:

$$
\mathscr{D}_{2}(x)=\left\|X_{1, i}-X_{2, j}\right\|_{2}
$$

Those 4 matrices are displayed in Figure 16 with the same colorbar. In this example, we can see that the error is always really small for the extremum point $B$ contrary to the other points. Nevertheless, they all have in common a 'line' representing the set 
of lags where the error is minimum. Those 'lines' are shown in Figure 17 and we can notice that they overlap. They describe the sets of lags $\left[\tau_{1}, \tau_{2}\right]$ to use that would guaranty a match of the 4 extremum points for the two signals. Thus, we can deduce a relationship between the sets of lags by performing a linear regression. We obtain that:

$$
\tau_{2}=3 \tau_{1}
$$

which is consistent with the dilation coefficient.
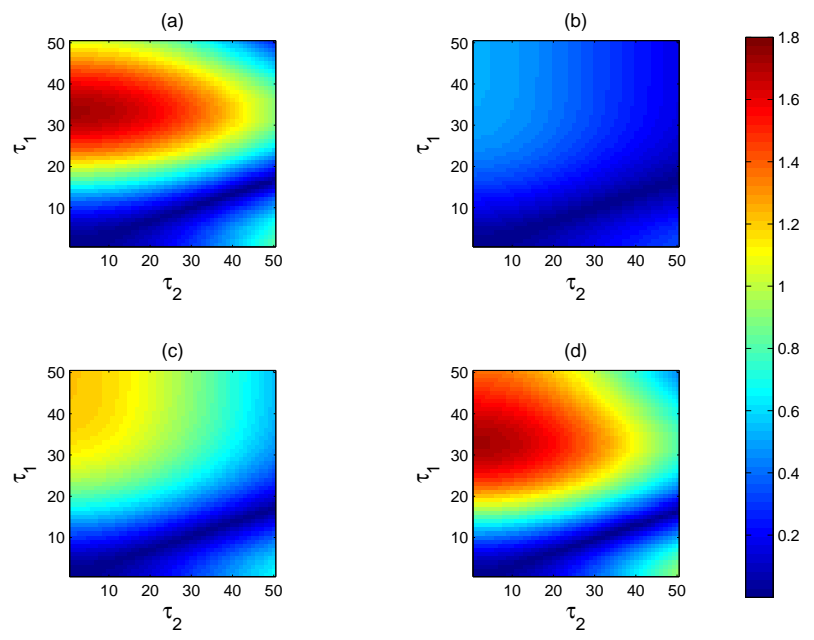

Fig. 16 The four matrices displaying the distance between extremum points of $s_{1}(n)$ and $s_{2}(n)$ 's phase space diagrams for different values of lags $\tau_{1}$ and $\tau_{2}$ : (a) $H_{A}$, (b) $H_{B}$, (c) $H_{C}$ and (d) $H_{D}$.

To validate this result, we draw $s_{1}(n)$ and $s_{2}(n)$ 's phase space diagrams by using the set of lags $\left[\tau_{1}=3, \tau_{2}=9\right]$ (Figure 18). As a matter of fact both phase space diagrams overlap perfectly.

This technique allows to highlight time-scale transformations. However, even if this technique enables to match extremum points having the same coordinates for certain couples of lags, we need to keep in mind that trajectories can still be different as the other coordinates are not considered. This is why it is interesting to monitor the area covered by the trajectory in the phase space which is the subject of the next subsection.

\subsection{Area calculation}

Previous subsection has shown that it was possible to define four reference coordinates for each phase space diagrams that allow to find matching correspondances, however, it is not enough to guaranty the invariance. The next idea consists in cal- 


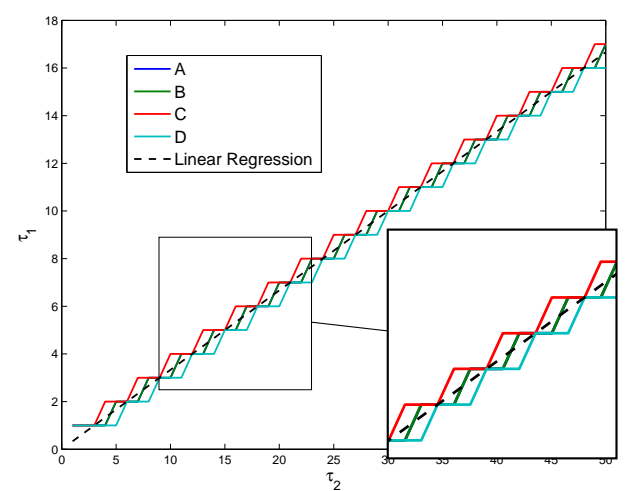

Fig. 17 Sets of lags $\left[\tau_{1}, \tau_{2}\right]$ that provide matches for the extremum points.

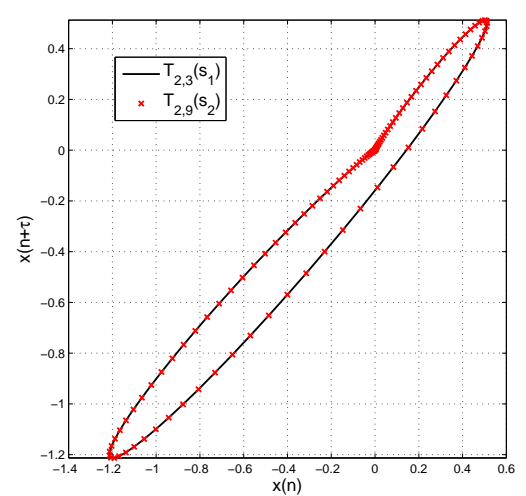

Fig. 18 Phase space diagrams of $T_{2,2}(s)$ and $T_{2,4}\left(s_{1}\right)$ respectively in black and red.

culating the area of the diagram to quantify the surface. To do so, the diagram is considered as a curve having at each instant $n$ polar coordinates $\left[\rho_{\tau_{k}}(n), \theta_{\tau_{k}}(n)\right]$. We then calculate the area $\mathscr{A}\left[s, \tau_{k}\right]$ covered by the phase space diagram calculated for $\tau=\tau_{k}$ :

$$
\mathscr{A}\left[s, \tau_{k}\right]=\int_{\theta_{\tau_{k}}(1)}^{\theta_{\tau_{k}}(N)} \rho_{\tau_{k}}^{2}(n)\left|d \theta_{\tau_{k}}(n)\right|
$$

We also define the matrix $A\left[s_{1}, s_{2}\right]$ defined as follows that enables to calculate the distance bewteen two signals $s_{1}$ and $s_{2}$ 's phase space diagram areas computed for different sets of lags $\left[\tau_{1}, \tau_{2}\right]$ :

$$
\begin{aligned}
A\left[s_{1}, s_{2}\right] & =\left\{a_{i, j}\right\}_{i, j \in\left[1, \ldots, \tau_{\max }\right]} \\
& =\left\{\left|\mathscr{A}\left[s_{1}, \tau_{i}\right]-\mathscr{A}\left[s_{2}, \tau_{j}\right]\right|\right\}
\end{aligned}
$$

It permits to find the sets of lags that offer similar areas for two phase space diagrams. This analysis can be complementary to the bounding box method to highlight time-scale transformation for instance.

In order to illustrate this concept, let consider the example presented previously that only presents a time-scale transformation. We first compute the area for $s_{1}(n)$ and $s_{2}(n)$ using $\tau=1, \ldots, 50$. The evolution of the area according to the lag is displayed for both signals in Figure 19. As we can see, $\mathscr{A}\left[s_{1}, \tau\right]$ presents a clear maxima for $\tau=17$, while $\mathscr{A}\left[s_{2}, \tau\right]$ does not have one. A maxima notifies a change on phase space diagrams that can as an example change its first eigenvector.

The computation of matrix $A\left[s_{1}, s_{2}\right]$ provides the sets of lags that offer matching areas. They are highlighted by a black dashed line in Figure 20. For $\tau_{1}=1, \ldots, 17$, we can see that this line is also a straight line that verifies the following equation:

$$
\tau_{2}=3 \tau_{1}
$$




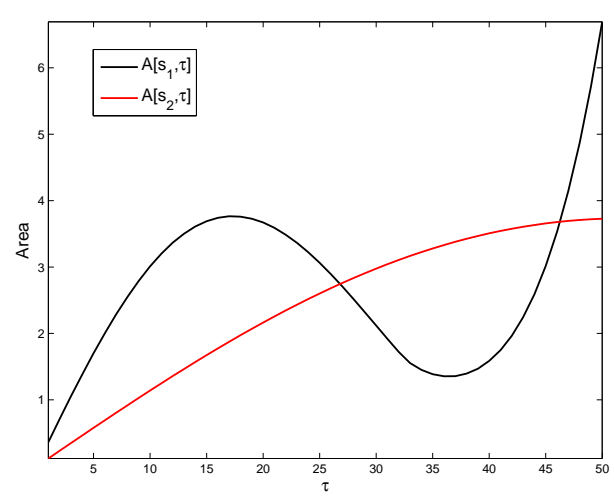

Fig. 19 Evolution of the two signals 's area according to the lag used to compute their phase space trajectories.

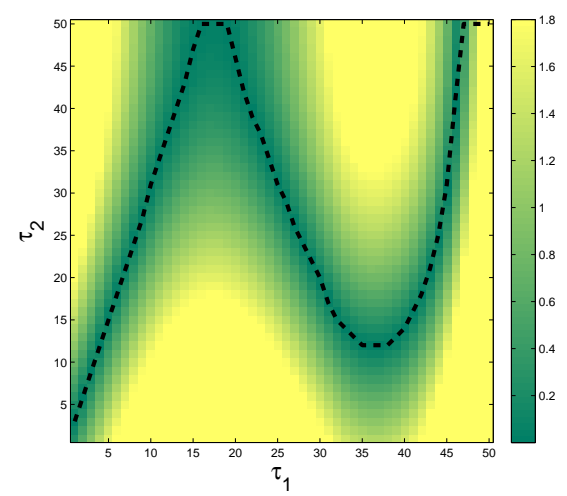

Fig. 20 The matrix $A\left[s_{1}, s_{2}\right]$ denables to calculate the distance bewteen two signals $s_{1}$ and $s_{2}$ 's phase space diagram areas computed for different sets of lags $\left[\tau_{1}, \tau_{2}\right]$.

This relationship confirms the dilation coefficient that has been used for the signals simulation.

This method is really usefull when coupled with the bounding box method. They enables to highlight time-scale transformations between signals.

\subsection{Polar coordinates analysis}

We have shown in Section 2 that amplitude changes can be enlightened from phase space diagrams. Indeed there exists a scale factor between two signals having an amplitude relationship. To highlight it, phase diagrams are turned into polar coordinates as shown in Figure 21. The assessment is that for a given polar angle, radius are directly connected by the amplitude coefficient.

Let consider $s_{1}(n)$ defined as in Eq. 2 and $s_{2}(n)$ defined as follows:

$$
s_{2}(n)=\frac{1}{\beta} s_{1}(\alpha n)
$$

with $\alpha=2$ and $\beta=1.4$, as an example. That is, $s_{2}$ is derived from $s_{1}$ by a double scale and amplitude modification.

Figure 15 and Figure 22 present temporal data and phase diagrams obtained for $m=2$ and respectively $\tau=8$ and $\tau=16$. Using this set of parameters, we know that the phase diagrams would be superposed if the signals's amplitudes were identical. However due to this difference they present a shape-invariant scale transformation.

We turn the cartesian coordinates into polar coordinates and draw the functions $\rho(n)=f(\theta(n))$ for the two signals that are shown in Figure 23. As we can see, both curves are similar and it is easy to imagine a linear relationship between them. 


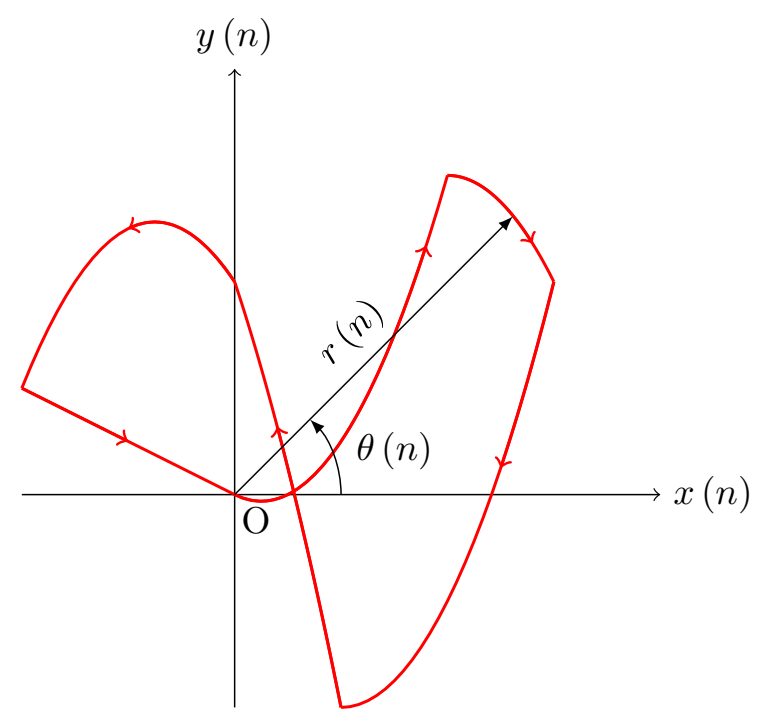

Fig. 21 Trajectory expressed in polar coordinate system. Each coordinates is represented by a radius and a polar angle.

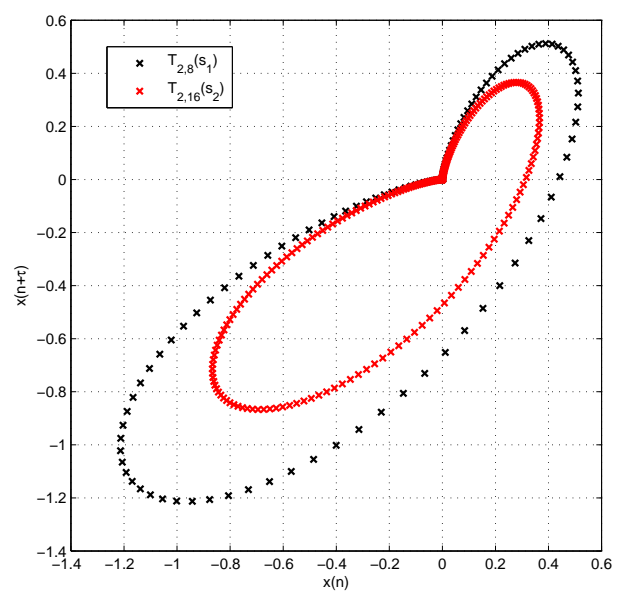

Fig. 22 Phase space diagrams of $T_{2,2}(s)$ and $T_{2,4}\left(s_{1}\right)$ respectively in black and red

However, for a given polar angle, there is not always a correspondance between the two curves (Figure 24). This is the reason why we propose an algorithm to match corresponding coordinates with respect to polar angles. 


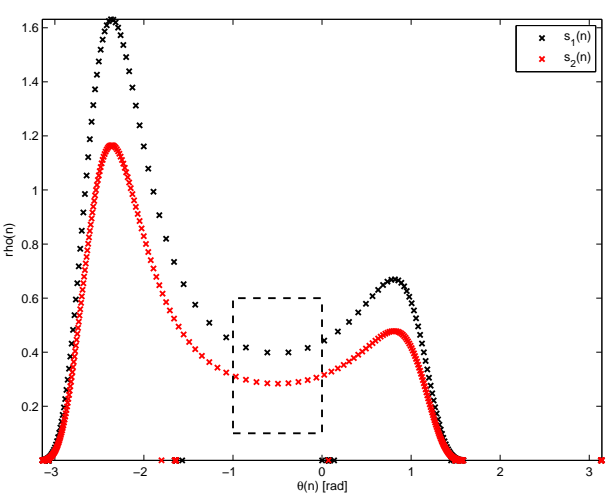

Fig. $23 \rho(n)=f(\theta(n))$ for $T_{2,2}(s)$ and $T_{2,4}\left(s_{1}\right)$ respectively in black and red

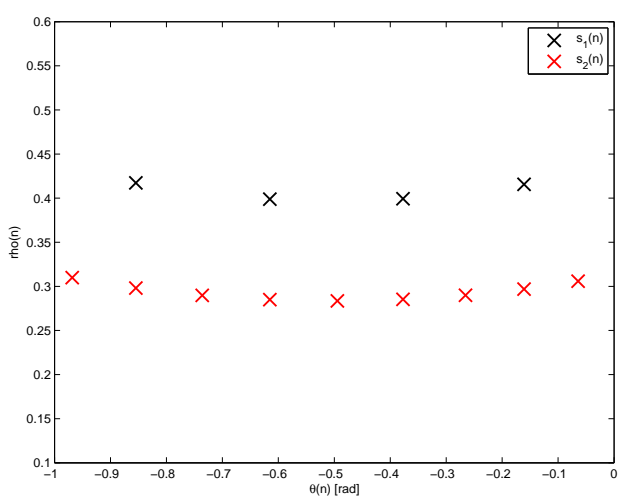

Fig. 24 Zoom in of Figure 23.

\section{Algorithm description:}

For each polar angle $\theta_{1}(n)$ of the first curve, the algorithm searches for the corresponding polar angle on the second curve $\theta_{2}(n)$. A match is enlightened if the two conditions are met:

$$
\left\{\begin{array}{l}
\theta_{2}\left(n_{1}\right)=\operatorname{argmin}\left|\theta_{1}\left(n_{0}\right)-\theta_{2}(n)\right| \\
\theta_{2}\left(n_{1}\right)<\varepsilon
\end{array}\right.
$$

where $n_{0}$ is the index of $\theta_{1}(n)$ we are looking for, $n_{1}$ is the corresponding index for $\theta_{2}(n)$ and $\varepsilon$ a threshold error that enables to discard certain associations that are not consistent.

The algorithm selects $M$ pairs of coordinates and calculates a ratio $r(i)$ defined for $i \in\{1, \ldots, M\}$ such as:

$$
r(i)=\frac{\theta_{1}\left(n_{0_{i}}\right)}{\theta_{2}\left(n_{1_{i}}\right)}
$$

Figure 25 presents the pairs of coordinates that have been selected by the algorithm and the associated ratio. In this example, we can see that the ratio is consistent along the entire polar angles interval which corroborates the idea of a shapeinvariant scale transformation. Moreover, the ratio is here equal to 1.4 which is the value of $\beta$.

We have seen in this example that it was possible to estimate a shape-invariant scale transformation. The same work can be done along an interval of polar angles that would highligh amplitudes changes on this interval. 

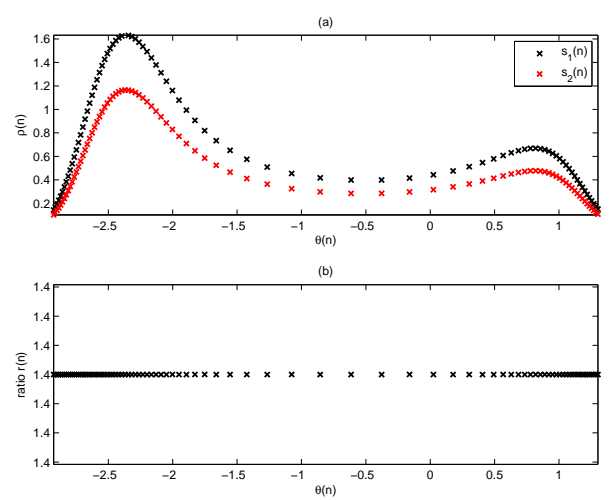

Fig. 25 (a) Pairs of coordinates that have been selected by the algorithm to calculate the amplitude modification known as $r(n)(\mathrm{b})$

\section{Application example}

In order to characterize transient modifications due to its propagation through a cable, we now conduct an experiment using the facilities existing in our lab. We generate a partial discharge (PD) on an electric cable whose ends $T_{1}$ and $T_{2}$ are connected to a data acquisition system. The PD source is respectively localized at $L_{1}$ and $L_{2}$ distances from the recording devices, as presented in Figure 26.The main idea is to estimate the relative propagation distances by comparing the relative deformations between the recorded signals. We note $s_{0}(n)$ the generated PD, $s_{1}(n)$ the signal recorded at $T_{1}$ and $s_{2}(n)$ the signal recorded at $T_{2}$.

$T_{1}$

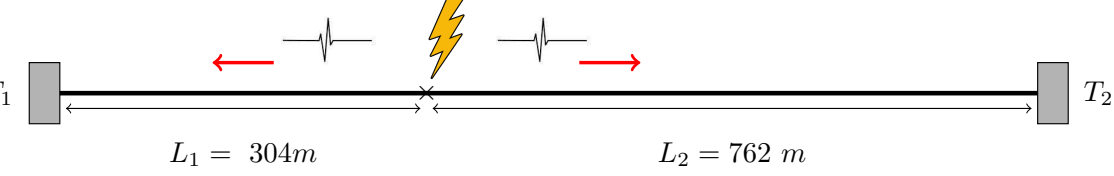

Fig. 26 Experimental outline

Time representation of emmitted and recorded signals are presented in Figure 27. As we can notice, signals recorded after propagation through the cable reels differ from the emitted one due to the propagation through the medium. They present nonlinear dilation and amplitude changes that are more or less stronger depending on the propagation range. Temporal changes are shown in Figure 28 where studied signals have been superposed and normalized.

What is interesting with this kind of signals is that we can easily visualize that a time-scale analysis would not be enough to characterize the modifications caused by the propagation. There are dilation and also a modification of the envelop of 


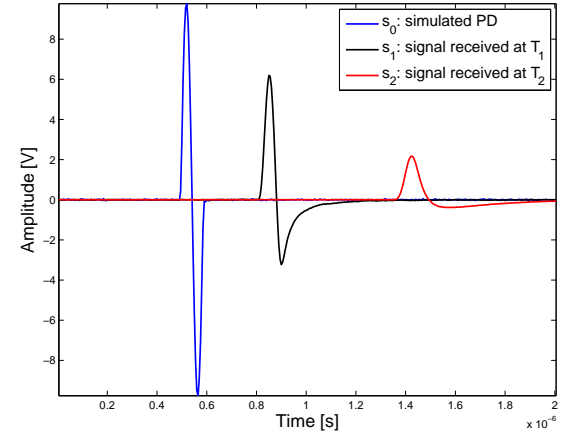

Fig. 27 Time representation of emmitted signal and recorded signals after propagation through the cable reels.

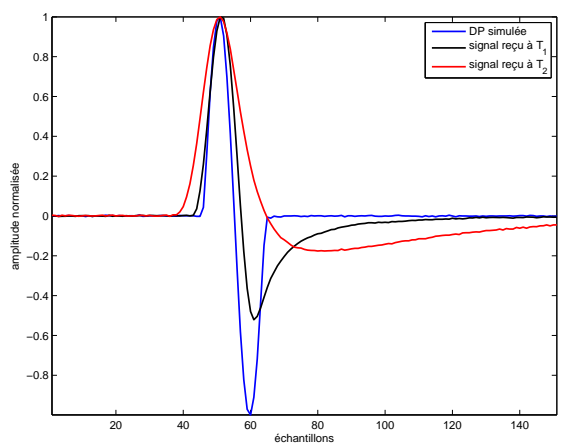

Fig. 28 Time representation of the superporsition of normalized signals that highligths dilation and amplitude changes.

the signal that would be difficult to analyze with a single type of mother wavelet function. This is shown by Figure 29 where a time-scale study is performed for the three signals using the Symlet mother wavelet. The results highly depend on the choice of mother wavelet used and for the three cases, even if it is easy to detect them, it is quite difficult to claim that they come from the same source as waveforms changes with the propagation. This is the reason why it is usefull to move forward MLPDA which is presented next.

(a)

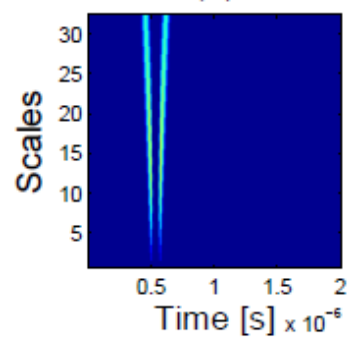

(b)

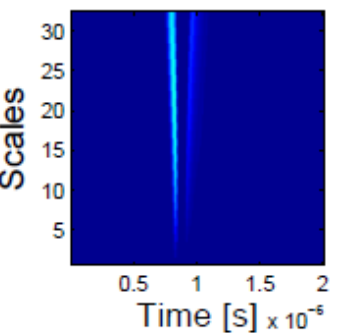

(c)

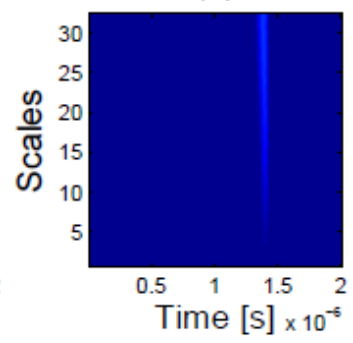

Fig. 29 Time-scale study using the symlet mother wavelet for (a) $s_{0}(n)$, (b) $s_{1}(n)$ and (c) $s_{2}(n)$.

To begin with, we need to normalize the signals by their maximal values, then compute their phase diagrams for $\tau=1, \ldots, 20$, search for the 4 extremum points $A, B, C$ and $D$, and finally compute the distance matrices $H_{A}, H_{B}, H_{C}$ and $H_{D}$ as presented in subsection 3.3. We then look for the sets of lags $\left[\tau_{1}, \tau_{2}\right]$ that minimize the distances between each extremum points.

Thus, after the computation, we can see that it is not possible to superpose the different $C$ and $D$ coordinates, which is coherent with the normalization of the signals by their maximum values. On the contrary, we can superpose the $A$ and $B$ coordi- 
nates. To illustrate this, we compute $T_{2,4}\left(s_{1}\right)$ and $T_{2,6}\left(s_{2}\right)$ shown in Figure 30, and $T_{2,5}\left(s_{1}\right)$ and $T_{2,8}\left(s_{1}\right)$ shown in Figure 31 . We can see that the top right parts of the two trajectories superpose well in the first case compared to the other one, even if the distances between $A_{1}$ and $A_{2}$, and $B_{1}$ and $B_{2}$ are smaller in the second case. This result confirms that the bounding box method cannot be used alone to determine dilation relationship. It needs to be coupled with a verification of the trajectories and/or other methods. Nevertheless, according to Figure 30, we can conclude that there is a dilation coefficient equal to $\frac{\tau_{2}}{\tau_{1}}=3 / 2$ between the positive parts of $s_{1}(n)$ and $s_{2}(n)$.

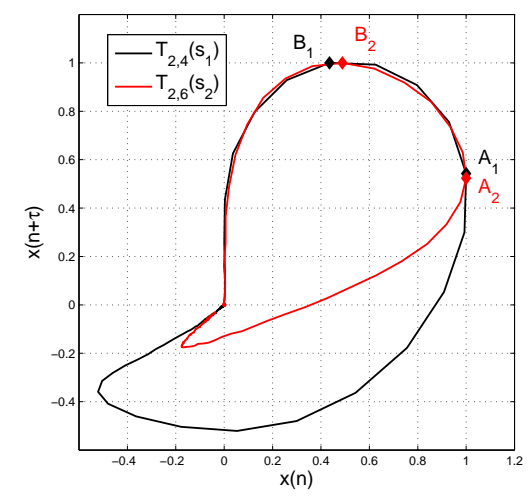

Fig. 30 Superposition of $T_{2,4}\left(s_{1}\right)$ and $T_{2,6}\left(s_{2}\right)$ phase space diagrams. Even if the distances $d\left(A_{1}, A_{2}\right)$ and $d\left(B_{1}, B_{2}\right)$ are not minimal, the top right parts of the diagrams superpose well.

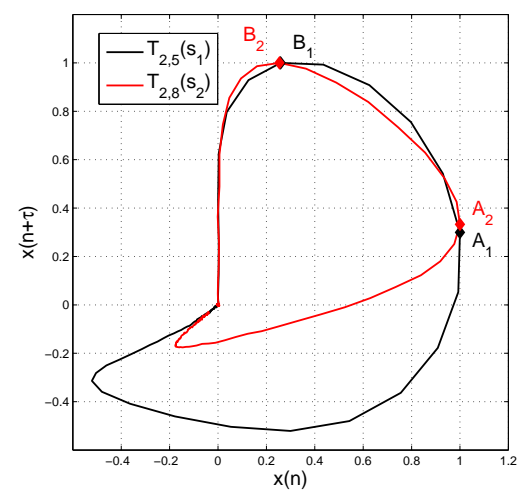

Fig. 31 Superposition of $T_{2,5}\left(s_{1}\right)$ and $T_{2,8}\left(s_{2}\right)$ phase space diagrams. The distances $d\left(A_{1}, A_{2}\right)$ and $d\left(B_{1}, B_{2}\right)$ are smaller than in the first case but the diagrams do not superpose well.

In order to quantify the time-scale coefficients obtained with MLPDA tool, we propose to define an estimated dilation coefficient obtained as follows. For the normalized signal's parts of interest, we calculate the number of samples where the signal's amplitude is higher than half the maximal value, i.e. 0.5 and then compute the ratio between the two numbers to obtain the estimated dilation coefficient. For positive part, we obtain a ratio $\tau_{1} / \tau_{2}$ equal to $8 / 12$, i.e. $2 / 3$ which corresponds to the coefficient we previously estimated.

As a conclusion, we can say that multi-lag tools have been used on partial discharge in order to characterize two signals received at two ends of cable reels. We have highlighted a time-scale transformation of the positive parts of the recorded data after propagation.Therefore, MLPDA tools have shown that they are promising to extract information from signals that suffer nonlinear modification. 


\section{Conclusions and perspectives}

In this article, we have shown in a first part that transients that were connected by time-shifted operator, time-scaling operator and amplitude modification, have invariant (or shape-invariant) phase diagrams if the lags were chosen wisely. We then proposed different methods to extract parsimonious parameters from each representation and combine all of them to highlight the named properties. So far, the developed methods provided good results for numerical examples as we showed in the last section.

Future axis of research can propose to extend this work to higher embedding dimensions. One possible idea could be to apply the same methods to the projections of phase diagrams onto the different planes of the phase space. More work should also be done to explore linear and nonlinear amplitude modifications.

\section{References}

1. J.-P. Eckmann, S. O. Kamphorst, and D. Ruelle, "Recurrence Plots of Dynamical Systems," Europhysics Letters (EPL), vol. 4, no. 9, pp. 973-977, Nov. 1987.

2. F. Takens, Dynamical Systems and Turbulence, Warwick 1980, ser. Lecture Notes in Mathematics. Springer Berlin Heidelberg, 1981, vol. 898.

3. N. Packard, J. Crutchfield, J. Farmer, and R. Shaw, "Geometry from a Time Series," Physical Review Letters, vol. 45, no. 9, pp. 712-716, Sep. 1980.

4. J. P. Zbilut and C. L. Webber, "Embeddings and delays as derived from quantification of recurrence plots," Physics Letters A, vol. 171, no. 3-4, pp. 199-203, Dec. 1992.

5. N. Marwan and J. Kurths, "Nonlinear analysis of bivariate data with cross recurrence plots," Physics Letters A, vol. 302, no. 5-6, pp. 299-307, Sep. 2002.

6. N. Marwan, "A historical review of recurrence plots," The European Physical Journal Special Topics, vol. 164, no. 1, pp. 3-12, Nov. 2008.

7. "Recurrence plots and cross recurrence plots," http://www.recurrence-plot.tk/, accessed: 201507-05.

8. C. Bernard, T. Petrut, G. Vasile, and C. Ioana, "Multi-Lag Phase Space Representations for Transient Signals Characterization," 22nd IEEE European Signal Processing Conference (EUSIPCO 2014), pp. 2115-2119, 2014. 